\title{
Research on Presence in Virtual Reality: A Survey
}

\author{
MARTIJN J. SCHUEMIE, M.Sc. ${ }^{1}$ PETER VAN DER STRAATEN ${ }^{1}{ }^{1}$ MEREL KRIJN, M.Sc., ${ }^{2}$ and \\ CHARLES A.P.G. VAN DER MAST, Ph.D. ${ }^{1}$
}

\begin{abstract}
Virtual Reality (VR) is starting to be used in psychological therapy around the world. However, a thorough understanding of the reason why VR is effective and what effect it has on the human psyche is still missing. Most research on this subject is related to the concept of presence. This paper gives an up-to-date overview of research in this diverse field. It starts with the most prevailing definitions and theories on presence, most of which attribute special roles for the mental process of attention and for mental models of the virtual space. A review of the phenomena thought to be effected by presence shows that there is still a strong need for research on this subject because little conclusive evidence exists regarding the relationship between presence and phenoma such as emotional responses to virtual stimuli. An investigation shows there has been substantial research for developing methods for measuring presence and research regarding factors that contribute to presence. Knowledge of these contributing factors can play a vital role in development of new VR applications, but key knowledge elements in this area are still missing.
\end{abstract}

\section{INTRODUCTION}

$\mathbf{V}$ IRTUAL REALITY (VR) IS QUICKLY becoming a serious tool for psychiatrists and therapists. However, even though research has shown VR to be effective in psychological therapy there is still much uncertainty about the reasons behind all this. For this, we need an understanding of the effect VR has on the human psyche. Current research on this subject is focussed primarily on the concept of presence.

The main goal of this paper is to investigate the current knowledge on presence to gain an understanding of the psychological mechanism underlying an experience in VR. This understanding is necessary for determining those aspects of the VR-system and the context in which it is used that contribute to an effective and efficient treatment.

Another goal of this article is to make a clear distinction between theory and empirical findings. Whenever possible, a description is made of the experiments supporting certain findings. The number of participants mentioned are those whose responses have been included in the statistical analysis underlying the findings. About statistical relationships between variables, the fact whether these are significant is reported, and a measure of the correlation is provided when it is available and independent of the used unit of measure.

After reviewing the most common definitions of presence and theories on the nature of presence this article will describe those phe-

\footnotetext{
${ }^{1}$ Department of Information Systems and Software Engineering, Faculty of Information Technology and Systems, Delft University of Technology, Delft, The Netherlands.

${ }^{2}$ Department of Clinical Psychology, Faculty of Psychology, University of Amsterdam, The Netherlands.
} 
nomena that are thought to be explained by presence. These "results of presence" are followed by a description of the various methods for measuring presence. To bridge the gap between presence and system and user characteristics, the research on factors that can cause presence is then reviewed.

VR is a technology that typically provides interaction and immerses the user's senses. This sets it apart from other technologies such as television or books. This paper deals primarily with presence in VR, even though this concept is also applied to nonimmersive and noninteractive media.

\section{ON THE NATURE OF PRESENCE}

In presence research several definitions and theories have been proposed; the prominent ones will be described here.

\section{Definitions}

The term "presence" is related to a wide field of research. Lombard and Ditton ${ }^{1}$ identified six different explications of presence that have been used in the literature: presence as

- social richness, the extent to which the medium is perceived as sociable, warm, sensitive, or personal when it is used to interact with other people;

- realism, the extent to which a medium can seem perceptual and/or socially realistic;

- transportation, the sensations of "you are there," "it is here," and/or "we are together";

- immersion, the extent to which the senses are engaged by the mediated environment;

- social actor within medium, the extent to which the user responds socially to a representation of a person through a medium; and

- medium as social actor, the extent to which the medium itself is perceived as a social actor (e.g., treating computers as social entities $\left.^{2}\right)$.

However, presence as discussed in literature related to immersive VR can most often be characterized by the concept of presence as transportation: people are usually considered "present" in an immersive VR when they report a sensation of being in the virtual world ("you are there"). The term co-presence or social presence is often reserved for the sense of being together in a virtual world ("we are together").

Sheridan ${ }^{3}$ makes another distinction. He emphasizes the difference between presence, that is the sense of being in a computer-generated world, and telepresence, the sense of being at a real remote location.

Heeter ${ }^{4}$ distinguishes between three different types of presence:

- personal presence, a measure of the extent to which the person feels like he or she is part of the virtual environment (VE);

- social presence, refers to the extent to which other beings (living or synthetic) also exist in the VE; and

- environmental presence, refers to the extent to which the environment itself acknowledges and reacts to the person in the VE.

Schloerb ${ }^{5}$ distinguishes two types of presence:

- subjective presence, the likelihood that the person judges himself to be physically present in the remote or virtual environment; and

- objective presence, the likelihood of successfully completing a task.

Schloerb's definitions of subjective and objective presence are completely empirical. Schloerb questions the value of subjective presence, because objective presence, the ability to work, should be the most important criteria for a VE.

An important distinction proposed by Slater and Wilbur ${ }^{6}$ is that between "presence" and "immersion":

- immersion: an objective description of aspects of the system such as field of view and display resolution.

- presence: a subjective phenomenon such as the sensation of being in a VE.

A less often used but often cited taxonomy is that of Zeltzer ${ }^{7}$ who argues that a VR system can be characterized by its: 
- autonomy, or the extent to which the VE is more than just passive geometry;

- interaction, the degree to which VE parameters can be modified at runtime; and

- presence, the measure for the number and fidelity of available sensory input and output channels.

Here, Zeltzer ${ }^{7}$ uses the term "presence" in a way that closely resembles the term "immersion" defined by Slater. ${ }^{6}$ Unfortunately, the word "immersion" is sometimes also used in a way closely resembling the subjective definition of presence (e.g., Bangay and Preston ${ }^{8}$ and Witmer and Singer $\left.{ }^{9}\right)$. In this article Slater and Wilbur's ${ }^{6}$ definitions of the terms "immersion" and "presence" will be used, unless stated otherwise.

\section{Theories}

In the literature, several theories on the nature of presence in immersive VR have been proposed. These will be described below.

Presence as non-mediation. In a discussion on the Presence-L Listserv during the spring of 2000 a general explication of presence was defined, which is still being refined today. The latest version to date (Sept. 2000) is:

\begin{abstract}
Presence (a shortened version of the term "telepresence") is a psychological state or subjective perception in which even though part or all of an individual's current experience is generated by and/or filtered through humanmade technology, part or all of the individual's perception fails to accurately acknowledge the role of the technology in the experience. Except in the most extreme cases, the individual can indicate correctly that $\mathrm{s} /$ he is using the technology, but at "some level* and to * some degree, ${ }^{*}$ her/his perceptions overlook that knowledge and objects, events, entities, and environments are perceived as if the technology was not involved in the experience. ${ }^{10}$
\end{abstract}

(Experience is defined as a person's observation of and/or interaction with objects, entities, and/or events in her or his environment; perception, the result of perceiving, is defined as a meaningful interpretation of experience.)

This explication emphasizes a dualism: part of the perception acknowledges that the expe- rience is mediated by technology, while another part does not. This is rarely explicitly stated in presence literature even though it is an essential aspect of the concept. People always know the experience is mediated, and, given the current state of technology, can always distinguish between mediated and direct stimuli. Nevertheless, at some level, the illusion of nonmediation can be perceived.

Exclusive presence. Slater et al. ${ }^{11}$ stress the participant's sense of "being there" in the virtual environment, and point out that a high sense of presence in a VE requires a simultaneous low level of presence in the real world and vice versa. Biocca ${ }^{12}$ states that "at one point in time, users can be said to feel as if they are physically present in only one of three places: the physical environment, the virtual environment, or the imaginal environment. Presence oscillates among these three poles." The level of presence experienced during an interval is dependent on the relative amount of time being present in the virtual world. ${ }^{13}$

Presence by involvement. Witmer and Singer 9 relate presence in part to the concept of attention: "presence may vary across a range of values that depends in part on the allocation of attentional resources."

- Involvement, a psychological state experienced as a consequence of focusing one's attention on a coherent set of stimuli or related activities and events.

- Immersion, defined as a psychological state characterized by perceiving oneself to be enveloped by, included in, and interacting with a VE. (This definition of immersion should not be confused with the oftenused definition of immersion as an objective measure as used in this article.)

Both involvement and immersion are thought to be necessary for experiencing presence. The authors state that by focusing attention a person will get more involved and will as a consequence experience a higher sense of presence. They call presence similar to the concept of selective attention, which "refers to the tendency to focus on selected information that 
is meaningful and of particular interest to the individual." 9

Ecological view. Another view of presence is based on the ecological theory of perception. ${ }^{14-16}$ Basic concepts of this approach are:

- The environment offers situated affordances. The term affordance was coined by Gibson $^{17}$ and is meant to describe the possibilities or opportunities that the environment (i.e., surroundings) of an animal, offers or affords the animal. For example, for a human, the ground affords walking, a chasm affords falling and hurting, an apple might afford eating, and a tiger affords being eaten. A particular affordance is dependent on both environment and animal. The surface of water in a ditch does not afford support or walking for humans, but it does for water bugs.

- Perception-action coupling. An organism perceives its environment in terms of its affordances, making perception dependent on possible action.

- Tools become "ready-to-hand." According to Heidegger ${ }^{16 a}$ using a tool precludes the user from possessing a stable representation of the tool. The user is no longer aware of the tool itself but only of the usefulness the tool has in whatever task is performed. ${ }^{16}$

Applying the concept of perception-action coupling to VR, one can conclude that the user will perceive the VR equipment in terms of what can be done with it (i.e., interacting in the VE). The mediating technology itself will eventually become "ready-to-hand," invisible to the user.

Zahorik and Jenison ${ }^{16}$ state that successfully supported actions in an environment will lead one to perceive oneself as existing in that environment, to a sense of presence. An action is said to be successfully supported when the result of that action is considered lawful: responses from the environment must be similar to those in the real-world environment in which our perceptual system evolved.

Social/cultural view. Other researchers agree with the key role of perceived possible interactions in presence but stress that "action is es- sentially social."18 Experiencing presence depends on whether the VE behaves and is constructed according to our cultural expectations and whether the VE is perceived and interpreted the same by others in the VE. ${ }^{19}$

Estimation theory. Sheridan ${ }^{20}$ in an attempt to combine the ecological perspective and the traditional rationalistic view, proposes the estimation theory. This theory supports the distinction between objective and subjective reality of the rationalistic approach, but states that we can never truly know objective reality, but are continuously making and refining a mental model which estimates reality, based on our senses and interaction with that reality.

Embodied presence. In a similar vein, Schubert et al. ${ }^{21}$ propose the embodied cognition framework by Glenberg as a means for explaining presence. A mental representation of the environment is made in terms of patterns of possible actions, based on perception and memory: "Presence is experienced when these actions include the perceived possibility to navigate and move the own body in the VE."

In predicting the outcome of actions, humans have the ability to suppress contributions of the current environment to conceptualization, thus explaining why we can experience presence in a VE despite sensing conflicting features of the real environment.

\section{Conclusions on the nature of presence}

The previous paragraph listed several definitions and theories on the nature of presence that do not necessarily contradict each other, although they can have different implications. To refine the concept of presence, better instruments for measuring presence are needed. Unfortunately, the way presence is measured depends on the theory used. Prothero et al. ${ }^{22}$ suggest that one way to escape this circularity is to use a converging approach: based on a simple theory, a measure can be developed which can be used to improve the theory, and so on. Already several research groups have attempted such an approach, as will be shown in the section on measures.

Kalawsky ${ }^{23}$ warns that "presence is a multi- 
dimensional parameter that is arguably an umbrella term for many inter-related perceptual and psychological factors." Most theories mentioned above attempt to explain presence in terms of several underlying factors. One common factor is that of attention. Also, most scholars relate presence to a mental model of the virtual reality with the (virtual) body in it.

In fact, Prothero et al. ${ }^{22}$ claim that "'Presence' and 'situation awareness' are overlapping constructs." Lackner and DiZio ${ }^{24}$ supply anecdotal evidence that when people have difficulty forming a mental model of a real (but nonterrestrial) space, they also report a loss of sense of presence.

\section{RESULTS OF PRESENCE}

For the concept of presence to be useful and applicable in practical situations it is important to understand the results or consequences of presence. This section will review the theories and empirical studies on the usefulness of presence, and thus its relationship to other constructs.

\section{Subjective sensation}

Almost every theory on presence refers to the subjective sensation of "being there" experienced and reported during immersion in a VE, and this sensation is in fact part of most definitions of presence. However, for instance, the explication statement from the Presence-L Listserv does not exclude a state where one does not have an explicit sensation of "being there" but still could be said to experience some form of presence.

This subjective sensation can apply to the environment currently being experienced, or to memories of past experiences. As Slater et al. ${ }^{25}$ note, a key result of presence is that a person remembers the $\mathrm{VE}$ as a place rather than a set of pictures.

\section{Task performance}

As Welch ${ }^{26}$ stated, "there is a pervasive belief that presence is causally related to performance... Despite the popularity of this notion, however, there is no solid evidence to support it." Welch continues by quoting Witmer in a personal communication about the review of several research projects: "significant correlation between presence and performance were the exception rather than the rule." Ellis ${ }^{27}$ even argues that for some tasks less presence might lead to better performance, for instance when a more abstract view of an environment is more helpful for completing the task.

Mania and Chalmers ${ }^{28}$ confirm that presence need not be related to task performance in an empirical study with three conditions: lectures were given on a specific topic in the real world, in a virtual classroom, and an auditory-only environment. In a between-subject design, 18 subjects were assigned to each condition. A preliminary analysis of the data was done by using a comparison of means and standard deviations and by applying the ANOVA method. Presence was found not to be correlated with the task performance of acquiring knowledge during the lecture.

Kim and Biocca ${ }^{29}$ however, in a study involving 96 subjects being exposed to an infomercial on TV, did find a weak but significant correlation between the part of their presence questionnaire labeled "departure" and both factual memory and average recognition speed for recognizing stills from the infomercial.

Slater et al..$^{30}$ and Steed et al. ${ }^{31}$ found a relationship between immersion and leadership. In a between-subject design with 30 subjects and 3 conditions (HMD and 2 types of desktop-VR systems), where subjects had to solve a riddle together, those in the more immersive conditions tended to evolve as the leaders of the group. However, no relationship between immersion and presence was found in this study.

\section{Responses and emotions}

Perhaps one of the most important consequences of presence is that a virtual experience can evoke the same reactions and emotions as a real experience.

Hodges et al. $^{32}$ in a between-subject experiment with 10 subjects on a wait-list and 10 subjects being treated for fear of heights in VR, showed that the subjects, who were all acrophobic, did show increased subjectively re- 
ported anxiety when confronted with height in the VE. They further showed that treatment in VR reduces acrophobia when compared to the waiting list. Later experiments around the world confirmed their findings, also for other phobias.

Regenbrecht et al. ${ }^{33}$ investigated the relationship between presence and fear of heights, both measured by questionnaires. In an experiment with 37 nonphobic subjects they did not find a significant correlation $(r=0.251, p>$ $0.10)$ between presence and fear. A regression analysis did show that presence was the best predictor of fear. Schuemie et al., ${ }^{34}$ in an explorative study with 10 subjects being treated for fear of heights, did find a significant correlation between fear and presence reported on questionnaires $(r=0.4461)$, but no significant correlation $(r=-0.325)$ between presence and reduction of acrophobia (also measured through questionnaires).

North et al. ${ }^{35}$ found that people can show signs of fear of public speaking when confronted with a virtual audience. Slater et al. ${ }^{25}$ in a between-subject study with 10 subjects and 2 conditions (positive and negative audience) showed that, in a regression analysis, presence tended to amplify the subject's response to the audience. In other words, people experiencing a higher level of presence were prone to report more negative reactions to a negative audience and more positive reactions to a positive audience.

When confronted with visual cues suggesting motion, a person will tend to correct for the perceived motion by adjusting their body posture. Freeman et al., ${ }^{36}$ in an experiment with 24 subjects, investigated the relationship between reported presence and postural responses. No significant correlation $(r=0.025)$ was found, however.

\section{Simulator sickness}

One problem associated with using VR is that it can cause nausea and dizziness, a phenomenon known as "simulator sickness." Witmer and Singer" found a significant negative correlation between simulator sickness reported on the Simulator Sickness Question- naire and presence measured using the Witmer and Singer questionnaire $(r=-0.426, p<$ 0.001).

In contradiction, Slater et al. ${ }^{37}$ found a positive correlation between simulator sickness and presence.

\section{Conclusions on results of presence}

Based on the current status of presence research, much uncertainty remains as to the usefulness of presence. Presence, when defined as a subjective sensation, can be a goal in itself for certain applications such as games and movies. Whether presence can contribute to better task performance is controversial based on the reported findings.

And although the usefulness of presence for emotional responses and phobia treatment seems to have more empiric evidence, this too is inconclusive. One problem here is that only weak evidence for a relationship between presence and emotional responses such as fear has been found, but no study has yet addressed the causality of this relationship. In other words, it is still unclear whether higher measured presence causes stronger emotional responses in a VE or the other way around.

\section{MEASURING PRESENCE}

Measures for presence are often based on the expected results of presence. A distinction can be made between subjective measures, requiring introspection by the subjects, and objective measures. Objective measures can further be divided into behavioral and physiological measures. These types of measurement will be reviewed below.

\section{Subjective measures: Questionnaires}

The most commonly used measures in presence research are based on subjective ratings through questionnaires. These will now be described in more detail, as well as some other subjective rating methods. This section will also present some of the components of presence and immersion that have been found through the use of these questionnaires com- 
bined with factor and cluster analysis methods.

As Witmer and Singer ${ }^{9}$ rightly stress, any measure of presence should be both reliable (i.e., only dependent on the characteristics under consideration), and valid (i.e., measuring what it intends to measure and measuring it well). More specifically, the authors emphasize that a measure or scale can have content validity, or "the coverage of the measured behavioral domain by the scale items" and construct validity, "the extent to which it can be said to measure a theoretical construct or trait." If available, these characteristics of the measurements will be mentioned here as well.

An advantage of questionnaires is that not only subjective sensations during the experience in a VE can be measured. Subjects can also be asked to describe the VE and their own physiological and behavioral responses, although these observations are of course less reliable because of their subjective nature.

Sometimes these questionnaires consist of a single question, for example, "I feel a sense of actually being in the same room with others when I am connected to a MOO." 38 To make the measure more reliable, often several questions are used. ${ }^{39,40}$ However, recently a more or less systematic approach has been taken to establish reliable and validated questionnaires. The most prominent ones will be described here.

Slater and colleagues. The questionnaire developed by Slater and colleagues is developed over a number of studies, ${ }^{41}$ and has received much attention in presence research. It is based on several questions, which are all variations on three themes: ${ }^{42}$

- the subjects sense of "being there";

- the extent to which the VE becomes more "real or present" than everyday reality; and

- the "locality," the extent to which the VE is thought of as a "place" that was visited rather than just a set of images.

These themes are directly derived from the research group's theory on the nature of pres- ence and are all strictly related to results of presence. The presence score is taken as the number of answers that have a high score.

Witmer and Singer. Based on their theory of involvement and immersion, and on previous empirical and theoretical research, Witmer and Singer ${ }^{9}$ determined several factors that are thought to contribute to a sense of presence:

- Control factors, the amount of control the user had on events in the VE.

- Sensory factors, the quality, number and consistency of displays.

- Distraction factors, the degree of distraction by objects and events in the real world.

- Realism factors, the degree of realism of the portrayed VE.

On their Presence Questionnaire (PQ), users can rate their experience in the VE according to these factors on questions with a 7-point Likert scale. The presence score is the sum of these ratings.

To validate this questionnaire, 152 subjects were asked to answer the questions after using a VE. The correlation between single items and the total score was investigated and most items showed a strong correlation. Items showing no significant correlation were deleted. However, as Slater ${ }^{43}$ pointed out, no correction was made for the fact that the item score already would be correlated with the total because the item itself is included in the sum. It is also interesting to note that the PQ is attempting to measure presence by measuring its causes, as evaluated by the user, and not its results.

The PQ was reduced by dropping items that did not contribute to its reliability. From a cluster analysis on data from this reduced PQ scale Witmer and Singer ${ }^{9}$ found three factors, which did not perfectly match the original factors, mentioned above. These factors, which regrouped items from the original factors, were labeled:

- Involved/Control-the control and responsiveness of a VE, and how involving a VE is.

- Natural-the naturalness of interactions 
and control of locomotion, and the consistency of a VE.

- Interface Quality-the amount of interference or distraction from task performance, and the participant's ability to concentrate on the tasks.

Calculations over multiple experiments indicated the reliability of the PQ to be 0.88 (Cronbach's $\alpha, n=152$ ). On the subject of content validity, the authors state that the PQ items were based on a review of the presence literature and "tap both aspects of presence: involvement and immersion." Witmer and Singer ${ }^{9}$ did a preliminary construct validation by checking the association with other variables and constructs such as simulator sickness, task performance, natural modes of interaction, spatial ability tests and relation to their Immersive Tendency Questionnaire measuring the tendency to become involved or immersed. The results of this validation were positive.

Igroup Presence Questionnaire (IPQ). Schubert et $a{ }^{44}$ constructed their IPQ by combining previous published questionnaires, among which were those of Witmer and Singer ${ }^{9}$ and Slater and colleagues, ${ }^{41,42}$ with a questionnaire from earlier research ${ }^{33}$ and some newly developed questions on technological and context variables. The resulting 75-item questionnaire was submitted to 246 volunteers, most of whom were male desktop-based-VR users. It should be noted that most entries were from gamebased VR systems, and only a small minority involved an HMD or a CAVE system. From a factor analysis eight factors were extracted; three of these were found to be concerned with presence itself, and five were identified as immersion factors. The presence factors, which entailed only subjective reports of how users experienced the VE, were:

- spatial presence (SP), the relation between the VE as a space and the own body;

- involvement (INV), the awareness devoted to the VE; and

- realness (REAL), the sense of reality attributed to the VE.

The immersion factors, the factors concerned with descriptions of the interaction of the user with the VE or with descriptions of the technological side of the VE, were:

- quality of immersion (QI), the sensory quality for richness and consistency of the multimodal presentation;

- drama (DRAMA), the perception of dramatic content and structures;

- interface awareness (IA), the awareness of interfaces that distract from the VE experience;

- exploration of $V E$ (EXPL), the possibility to explore and actively search the VE; and

- predictability (PRED), the ability to predict and anticipate what will happen next.

The authors contend that the factor analysis provides supporting evidence for a distinction between reports on subjective experiences-the presence factors-and reported evaluations of the technology-the immersion factors. Furthermore, the two factors spatial presence (SP) and involvement (INV) support the distinction between a spatial-constructive and an attention component. This distinction was postulated earlier by Witmer and Singer ${ }^{9}$ and was also derived by the authors from the Embodied Presence Model. Finally, Schubert et al. ${ }^{44}$ state that the two factors SP and INV together load on a first second-order factor, which thus might be a general presence factor. Though not predicted by the model, and to the authors' surprise, a third factor ("realness") also loaded on this general presence factor. Schubert and colleagues ${ }^{44}$ calculated the internal consistency of IPQ over two studies to be 0.85 and 0.87 (Cronbach's $\alpha ; n=264, n=296$ ).

Kim and Biocca. Kim and Biocca ${ }^{29}$ constructed a questionnaire with eight items based on theory and questionnaires by other authors. Ninety-six subjects filled in the questions after having been exposed to an infomercial on TV. A factor analysis found two factors, which were labeled using a metaphor of transportation:

- arrival, being present in the mediated environment; and

- departure, not being present in the unmediated environment.

The arrival factor included items related to feeling one had arrived in another world than 
the real one (the world of the infomercial); the departure factor included items relating to the feeling one had never left the real world.

ITC Sense Of Presence Inventory (ITC-SOPI). The questionnaires mentioned so far were designed with certain media in mind, such as immersive VR. Lessiter et al. ${ }^{45}$ attempted to create a measure that should apply across a range of media; for example, also to television and cinema. For their ITC-SOPI questionnaire 63 items were generated which were thought to have relevance to the concept of presence. All items had a 5-point Likert scale. The questionnaire was administered to 604 people following their experience of a mediated environment. Factor analysis found four factors:

- Physical Space: for example, "I had a sense of being in the scenes displayed," "I felt I was visiting the places in the displayed environment," "I felt that the characters and/or objects could almost touch me."

- Engagement; for example, "I felt involved (in the displayed environment)," "I enjoyed myself," "My experience was intense."

- Naturalness; for example, "The content seemed believable to me," "I had a strong sense that the characters and objects were solid," "The displayed environment seemed natural."

- Negative effects; for example, "I felt dizzy," "I felt disorientated," "I felt nauseous."

Eventually 44 items were retained, which loaded on one of these factors. The similarity between the first three factors and the factors found by Schubert et al. ${ }^{44}$ is, as noted by the authors, striking. Lessiter and colleagues ${ }^{45}$ calculated the internal consistency coefficients for each of the four factors and found alphas ranging from 0.94 (Physical Space) to 0.76 (Naturalness). The authors did a preliminary validation of their questionnaire by comparing its results to results they obtained by using comparable (but not identical) questions from the Slater et al. questionnaire. The results showed that both questionnaires load onto the same factors and can discriminate between different media. Further studies are planned to directly compare the ITC-SOPI with Slater et al.'s ${ }^{42}$ questions. The authors also computed scale scores for each factor for the different media across which data were collected. They found that the scores for the factors were correlated to the media format in a predictable way. For instance, the factor Physical Space showed sensitivity to media format.

Lombard and Ditton. Lombard and Ditton ${ }^{2}$ are also creating a cross-media presence questionnaire. Participants were assigned to one of two conditions: high or low presence. The high presence condition involved a 3D IMAX movie, the low presence condition a 12-inch black and white television. A 103-item questionnaire was developed based on items used by other authors. So far, 307 subjects completed the high presence condition. The experiment is still ongoing. In a factor analysis of the preliminary results the following factors are found:

- immersion relates to the sense of immersion, involvement and engagement in the mediated environment;

- parasocial interaction relates to interacting with other people in real time in the mediated environment;

- parasocial relationships concern feelings of friendship, etc., toward people in the VE;

- physiological response concerns, amongst others, simulator sickness;

- social reality relates to how likely the events are to occur in reality;

- interpersonal social richness relates to how well the user could observe interpersonal communication cues; and

- general social richness relates to items such as unemotional/emotional, unresponsive/ responsive, impersonal/personal.

Conclusions on questionnaires. Presence questionnaires are all originally developed from certain theoretical views on the concept of presence. This basis determines the scope of the questionnaires as well as the relevant application domain, such as immersive VR or TV.

In turn, the measures are used to refine the theories on which they are based. Techniques such as factor analysis show that there are several major components in reported subjective sensations on presence, and that these compo- 
nents are related. However, the relationships between these components and the concept of presence may not be assumed as given, and depends in part on the definition of presence used. Also, the components found depend very much on the original scope of the study and its related questionnaire. This is obvious for instance in the noninteractive design of Lombard's experiment. ${ }^{2}$ Another example is the Kim and Biocca questionnaire ${ }^{29}$ with its limited number and diversity of questions, which therefore was limited in the number and diversity of factors found.

Subjective measures can be prone to errors. For instance, prior experience in rating stimuli on other aspects such as interest and 3D-ness affected the subsequent rating of presence in a study involving 72 subjects. ${ }^{46}$ Similar results were found by Welch et al. ${ }^{47}$ in a preliminary study, where the order in which the VEs were presented was found to have a main effect on the relative presence ratings of these VEs when compared to each other.

Usoh et al. ${ }^{41}$ subjected two questionnaires to a reality-test in a between-subject design; 10 subjects completed a search task in reality, 10 completed the task in a VE. If the questionnaires measured correctly, subjects should report a greater sense of presence in reality than in the VE. However, subjects did not report significantly different presence ratings on the Witmer and Singer PQ for the two conditions. ${ }^{9}$ The questionnaire by Slater and colleagues did find a marginal but significant difference. Interestingly, Mania and Chalmers ${ }^{28}$ when using the latter questionnaire, did find presence to be "much higher and significantly different" for a real situation compared to two virtual ones (18 subjects in the real condition, 18 subjects in an immersive $\mathrm{VE}$, and 18 subjects in an audio-only VE).

\section{Other subjective measures}

Continuous measure. Instead of administering a questionnaire only after a virtual experience, IJsselstein and de Ridder ${ }^{48}$ proposed a continuous measure of presence during the experience. In a study involving 24 subjects, a hand-operated slider could be used to indicate the level of presence experienced at that mo- ment. Analysis showed that rated presence increased with the addition of stereoscopy and of motion cues.

Presence counter. Based on their theory that individuals are, at one moment in time, either completely present in the real or in the virtual world, Slater and Steed ${ }^{13}$ proposed a presence counter which measures the number of transitions in presence. Because the action of reporting such a transition requires a person to feel present in the real world, only transitions from the virtual to the real world can be recorded. Based on a simple Markov Chain model, this counter is used to estimate the relative time the person was present in the virtual world. In a between-subject study with 18 participants significant correlations were found between body movement and presence $\left(r^{2}=0.38\right)$ and between presence measured through the presence counter and through a traditional questionnaire $\left(r^{2}=0.32\right)$.

Focus group exploration. To gain more qualitative insight into the concept of presence, Freeman and Avons ${ }^{49}$ used Focus Group Exploration. This method requires small groups to discuss a topic, in this case people's experience while watching stereoscopic TV. Results show that non-experts describe sensations of presence, and are relating presence to involvement, realism, and naturalness. Heeter ${ }^{4}$ applied a similar approach, questioning users after they had used immersive VEs.

\section{Objective measures: Behavioral}

As mentioned in the section on results of presence, people tend to respond to mediated stimuli as if it were unmediated when they experience a high level of presence. Examining people's reaction to mediated stimuli could provide an objective measure of presence.

Sheridan ${ }^{50}$ proposes measuring reflex responses, such as automatically trying to catch a ball or trying to avoid a rapidly approaching object. As mentioned before, Freeman et al. ${ }^{36}$ attempted to use postural response as a measure for presence but found no significant correlation between this measure and reported presence. 
Prothero et al. ${ }^{22}$ propose so-called "class A" measures of presence, which measure subjects' responses to virtual cues when subjects are also presented with conflicting real cues. Slater et al. ${ }^{42}$ used such a method: subjects were shown a radio in reality and then had to put on an HMD that showed a VE with a radio at the same location as the real one. During the experiment, the real radio was moved and turned on. The subject was required to point at the radio when this happened. A high degree of presence would lead to the subject pointing at the virtual radio rather than the real one. In the study, involving eight subjects, a significant correlation was found between this objective measure and a presence questionnaire.

O'Brien et al. ${ }^{19}$ used an ethnographic approach to study users' behavior, analyzing human to human interaction in multi-user VEs. This led to more qualitative insight in the nature of presence, linking presence to the concept of intersubjectivity (i.e., the things people know in common).

\section{Objective measures: Physiological}

Sheridan ${ }^{3}$ warns that "'Presence' is a subjective sensation, much like 'mental workload' and 'mental model' - it is a mental manifestation, not so amenable to objective physiological definition and measurement." Meehan, ${ }^{51}$ however, attempted to measure presence using heart rate, skin temperature, and skin conductance in an experiment where 10 subjects were exposed to a virtual height situation in a sophisticated immersive VE. Although results regarding heart rate and skin temperature were inconclusive due to noise in the heart rate measurement and slow change in skin temperature, a correlation was found between skin conductance and presence as measured using the questionnaire by Slater and colleagues. These results tend to be supported by the findings of Wiederhold et al., 52 who performed a withinsubject experiment with five subjects, one diagnosed as having fear of flying. During exposure to an airplane simulator on either a screen or using an HMD, skin conductance was found to be significantly higher for the HMD condition, which also generated the highest presence ratings on a presence questionnaire.
It is important to note that skin conductance, like most physiological measures, is related to arousal and not directly to presence. In the two experiments mentioned above, higher presence was related to a decrease in skin conductance level, indicating increased arousal. For illustration: Wilson and Sasse ${ }^{53}$ report that at lower video frame rates skin conductance is lower, indicating stress and arousal. Lower frame rates are, however, associated with lower presence as described in the section on causes of presence.

\section{Conclusions on measures}

Measuring presence is done almost exclusively via questionnaires, using them to refine the theories on presence and, surprisingly, to validate objective measures. A reason for this is the fact that presence theory is still being developed, and questionnaires offer rich feedback required to aid in the understanding of the phenomenon being measured.

\section{CAUSES OF PRESENCE}

Much research has been devoted to finding factors that contribute to presence. Several researchers constructed different categorizations of these factors.

1. Slater and Usoh ${ }^{54}$ :

- High quality, high resolution information.

- Consistency across all displays.

- Interaction with environment.

- Virtual body, the representation of the user's body in the VE.

- Effect of action should be anticipated.

2. Witmer and Singer ${ }^{9}$ :

- Control factors, the control the users has.

- Sensory factors, the richness of the displayed information and consistency across displays.

- Distraction factors, how much the user is distracted from the VE.

- Realism factors, pictorial and social realism of the VE.

3. Sheridan ${ }^{3}$ :

- Extent of sensory information. 
- Control of relation of sensors to environment.

- Ability to modify physical environment.

\section{Lombard and Ditton ${ }^{1}$ :}

- The form in which the information is presented.

- The content of the information.

- User characteristics.

\section{Steuer ${ }^{55}$ :}

- Vividness refers to the ability of a technology ${ }^{55}$ to produce a sensorially rich mediated environment.

- Interactivity refers to the degree to which users of a medium can influence the form or content of the mediated environment.

- User characteristics refers to the individual differences in users.

In the following paragraphs an overview is provided of the empirical research concerning these factors. For this, a categorization is used closely resembling that of Steuer. ${ }^{55}$ This choice was made based on the following argument: first of all it is important to make a distinction between characteristics of the system and of the user, since the former are in control of the designer while the latter must be accepted as given. Furthermore, as described in the section on presence theory, several authors ascribe a unique role to interaction as a key element of presence, arguing for paying special attention to interactivity factors.

\section{Vividness}

According to Steuer, ${ }^{55}$ vividness refers to the ability of a technology to produce a sensorially rich mediated environment. In this article, this category has a broader meaning than Steuer originally intended and also includes factors related to the content of a VE, since these can be closely related to sensory fidelity of the display used for showing this content.

Several empirical studies have found a relationship between aspects of VE vividness and presence. The results of these studies have been summarized in Table 1 and are further elaborated in the following paragraphs.

Axelsson et al. ${ }^{56}$ found subjects to have a sig- nificantly higher sense of presence in a CAVE when compared to desktop VR; 44 subjects were required to solve a three-dimensional puzzle together in VR, half of them using a CAVE-system while the other half used desktop VR. Presence was measured using a single question.

Barfield et al. ${ }^{57}$ reported a significant effect for update rate in a study involving eight subjects in a within-subject design. The six conditions were based on combinations of two variables: type of input device and update rates of 10, 15, and $20 \mathrm{~Hz}$. Presence was measured mainly through one question.

Prothero and Hoffman ${ }^{58}$ found a weak but significant effect for Field Of View (FOV). The study involved 38 subjects in a within-subject design with 2 conditions: $60^{\circ} \mathrm{FOV}$ and $105^{\circ}$ FOV. Presence was measured using a 5-item questionnaire.

Kim and Biocca, ${ }^{29}$ however, did not find any effect for FOV in an experiment involving 96 subjects, exposing them to an infomercial on TV in a between-subject experiment with 6 conditions based on 2 variables: FOV and illumination of the real environment. FOVs were chosen of $9.8^{\circ}, 21.5^{\circ}$, and $33.7^{\circ}$. Presence was measured using the Kim and Biocca ${ }^{29}$ questionnaire. Illuminating the real environment, making it more distracting, also had no significant effect on presence.

Prothero et al. ${ }^{59}$ reported a significant effect for foreground/background manipulations: the screen was masked either near the eye or near the computer screen. In this within-subject experiment 39 subjects participated with the two conditions of foreground or background masking. Participants reported higher presence for the eye masking on a 5-item questionnaire.

Hendrix and Barfield ${ }^{40}$ found significant effects for Geometric Field Of View (GFOV, the view-angle represented on the screen, which can be independent of the screen size or regular FOV) and stereoscopy in a study with a within-subject design involving 12 subjects. Subjects participated in three consecutive experiments in which one of three variables was manipulated: stereoscopy, head tracking, and GFOV of $10^{\circ}, 50^{\circ}$, or $90^{\circ}$. Presence was measured using a 2-item questionnaire. 


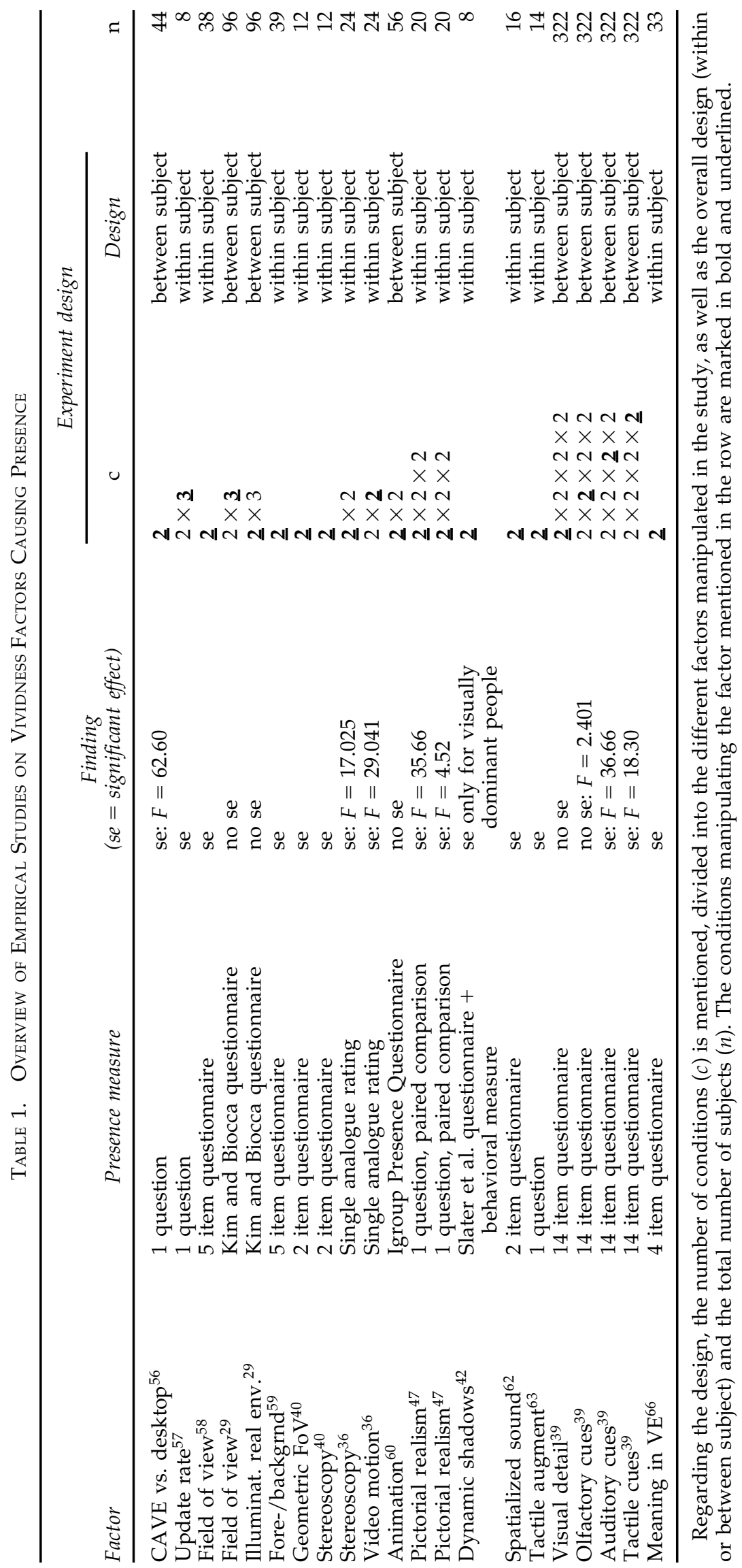


Freeman et al. ${ }^{36}$ also found a significant correlation between stereoscopy and presence, as well as between presence and video motion; 24 subjects in a within-subject design were exposed to 4 conditions based on 2 variables: stereoscopy and moving or still images. Presence was measured using an analogue rating scale. Subjects were required to mark their level of presence with a dot. The distance between the dot and the bottom line was taken as a measure for presence.

Schubert et al. ${ }^{60}$ found no significant effect for animation in the form of doors opening and comic-strip-like shoes walking in and out. The between-subject study involved 56 subjects and 4 conditions based on 2 variables: animation and self-movement. Presence was measured using the IPQ.

Welch et al. ${ }^{47}$ reported a significant effect for pictorial realism. In a study with 20 subjects and 8 conditions in a within-subject design the method of paired comparison was used, a procedure where the subjects are exposed to two VEs and are required to indicate which generated a higher sense of presence. Combinations of three variables determined the conditions: pictorial realism, interactivity, and the order in which the 2 VEs were presented. Presence was measured using a single question. A second almost similar study confirmed these findings. The conditions for this experiment were determined by pictorial realism, feedback delay, and order.

Slater et al. ${ }^{42}$ reported on the effect of dynamic shadows (i.e., shadows that change in real time when the scene changes). In a withinsubject study eight participants were exposed to VEs with or without dynamic shadows. Presence was measured using the Slater and colleague's questionnaire ${ }^{42}$ and a behavioral test as described in the section on measures. In general, the shadows did not have a significant effect on presence, unless the person was visually dominant (see the section on user characteristics). Note however, that this subgroup has an even smaller population than the already small sample in the entire study, and that including the dynamic shadows reduced the speed of the VE dramatically, possibly creating artifacts in the measurements.

Based on reports from suddenly deafened adults, Gilkey and Weisenberger ${ }^{61}$ conclude that "hearing may play a special role in perception, which strengthens the coupling between the observer and the environment, and enhances the sense of presence."

Hendrix and Barfield ${ }^{62}$ reported a significant effect of spatialized sound when compared to no sound or even to nonspatialized sound; 16 subjects were used in 2 within-subject experiments. The first experiment compared no sound with spatialized sound, the second compared spatialized sound with non-spatialized sound. Presence was measured using two questions.

Hoffman et al. ${ }^{63}$ reported a strong significant effect for tactile augmentation; 14 subjects participated in a within-subject design with 2 conditions. In one condition the subjects could see a ball; in the second condition they could also touch it because a real ball was placed in exactly the same position as the virtual one. Presence was measured using one question.

Dinh et al. ${ }^{39}$ performed an extensive study with 322 participants in a between-subject design. There were 16 conditions, based on combinations of 4 variables: high or low visual detail, olfactory cues, auditory cues, and tactile cues. Presence was measured using a 14-item questionnaire. An ANOVA showed significant main effects for auditory and tactile cues. Olfactory cues and higher visual detail did not have a significant effect on presence. No interactions were found between the variables, suggesting additional sensory cues work in a simple additive fashion on one's sense of presence.

Hoffman et al. ${ }^{66}$ found that when chess pieces in a VE were positioned in a meaningful way, this contributed to a significantly higher sense of presence for experienced chess players in an experiment involving 33 subjects of 4 categories: nonchess players, weak players, strong players, and tournament-level chess players.

\section{Interactivity}

Steuer ${ }^{55}$ defines interactivity as "the extent to which users can participate in modifying the form and content of a mediated environment in real time." The ecological theories especially place a large emphasis on the role of interac- 
Table 2. Overview of Empirical Studies on Interaction Factors Causing Presence

\begin{tabular}{|c|c|c|c|c|c|}
\hline \multirow[b]{2}{*}{ Factor } & \multirow[b]{2}{*}{ Presence measure } & \multirow{2}{*}{$\begin{array}{c}\text { Finding }(s e= \\
\text { significant effect })\end{array}$} & \multicolumn{2}{|c|}{ Experiment design } & \multirow[b]{2}{*}{$\mathrm{n}$} \\
\hline & & & c & Design & \\
\hline Interaction $^{47}$ & 1 question, paired comparison & se: $F=14.00$ & $2 \times \underline{2} \times 2$ & within subject & 20 \\
\hline Feedback delay ${ }^{47}$ & 1 question, paired comparison & se: $F=30.94$ & $2 \times \underline{2} \times 2$ & within subject & 20 \\
\hline Input device type $e^{57}$ & 1 question & no se & $\underline{2} \times 3$ & within subject & 8 \\
\hline Body movement 64 & Slater et al. questionnaire & se & $\underline{2} \times 2$ & between subject & 20 \\
\hline Head tracking ${ }^{40}$ & 2 item questionnaire & se & $\underline{2}$ & within subject & 12 \\
\hline Head tracking 60 & Igroup Presence Questionnaire & se & $2 \times 2$ & between subject & 56 \\
\hline Illusory interaction ${ }^{60}$ & Igroup Presence Questionnaire & se only on SP & 2 & between subject & 26 \\
\hline Social cues ${ }^{65}$ & Questionnaires & no se & $\underline{2}$ & between subject & 48 \\
\hline
\end{tabular}

Regarding the design, the number of conditions $(c)$ is mentioned, divided into the different factors manipulated in the study, as well as the overall design (within or between subject) and the total number of subjects ( $n$ ). The conditions manipulating the factor mentioned in the row are marked in bold and underlined.

tion in creating presence. The results of studies relating interactivity to presence have been summarized in Table 2. Further details are provided in the following paragraphs.

Welch et al. ${ }^{47}$ reported a significant positive effect for interaction in general and a negative effect for feedback delay in the two experiments already described in the previous section on vividness. Interaction was manipulated by either letting the subject drive a car or be a passive observer. Feedback delay, the delay between a user action and the response of the display to that action, was set at either the minimum possible with the equipment (which was 200-220 msec) or at an additional $1.5 \mathrm{sec}$.

Barfield et al. ${ }^{57}$ did not find a significant effect for type of input device when comparing a 3 Degrees-Of-Freedom (DOF) joystick with a 3 DOF space mouse in the study already described in the section on vividness.

Slater et al. ${ }^{64}$ found a positive significant effect for body movement; 20 subjects were used in a between-subject design with 4 conditions. The VE portrayed an area with plants, some of which had leaves with discolored undersides. All subjects were given the task of counting the diseased plants. For half the subjects, the plants were of similar height and could easily be inspected while looking at eye height in standing position; for the other half there was greater variance in the height of the plants and the subjects had to move their bodies to see the undersides of the leaves. Half of the subjects were given the extra task of also remembering the location of the diseased trees.
Hendrix and Barfield ${ }^{40}$ reported a highly significant effect for head tracking in an experiment already described in the section on vividness.

Schubert et al. ${ }^{60}$ performed two studies. The first was already described in the section on vividness and also showed a significant effect for head tracking. The second experiment, with 26 subjects in a between-subject design, had 2 conditions: either the participants were told that the animations in the VE were independent of their actions or they were told the animations responded to the user's actions (it was not said in which way). This illusory interaction did not have a significant effect on overall presence. It did however have a small but significant effect on Spatial Presence (see the IPQ).

A special case of interaction in VEs is interaction between users. A distinction is made between presence and social presence or co-presence: the sense of being together in the virtual world. Social presence is thought to be a part of overall presence. This is supported by at least two studies. Slater et al. ${ }^{30}$ found a significant positive correlation between presence and co-presence in an experiment with 30 subjects. Similarly, Thie and van Wijk ${ }^{65}$ found a significant relationship between presence and copresence $(r=0.458)$ in an empirical study with 48 subjects using desktop VR. In this experiment, social cues, however, were found to have no significant effect on either social presence or presence. Social cues in one condition were limited because users could not pick their own Avatar or nickname, nor could they make ges- 
tures. In the second condition this was made possible.

However, in the study by Axelsson et al. ${ }^{56}$ described in the section on vividness, presence and social presence were not found to be related. Subjects reported higher presence in the CAVE system, but not higher social presence.

\section{User characteristics}

Different individuals when being confronted with the same VE can still experience different levels of presence because of individual differences. Steuer ${ }^{55}$ mentions the willingness to suspend disbelief which is necessary for experiencing presence. Witmer and Singer ${ }^{9}$ have constructed the Immersive Tendencies Questionnaire (ITQ). Cluster analysis of data filled in by 152 subjects revealed three subscales:

- involvement, or the propensity of subjects to get involved passively in some activity, such as reading a book;

- focus, or the ability to concentrate on enjoyable activities and block out distraction; and

- games, or the frequency with which the subject plays games and the level of involvement in these games.

Out of four experiments, only two found a significant correlation between the ITQ and the PQ. Combining the data across experiments showed a significant correlation between ITQ and PQ.

Slater and Usoh ${ }^{54}$ use the therapeutic technique known as NeuroLinguistic Programming (NLP) to characterize the user's psychological perceptual system. The NLP model claims that subjective experience is encoded in terms of three main representation systems: visual (V), auditory (A), and kinesthetic (K), and that people have a tendency to prefer one system over the others. Furthermore, when a person represents a memory they tend to choose one of three perspectives: first, second (from another person's view), or third (from an abstract, nonpersonal view) person. Determining someone's preferred representational system and perspective can be done by analyzing their choice of words. A between-subject study with 17 subjects and 2 conditions (with or without a virtual body) was performed. A regression model showed positive significant correlation between presence and use of the visual representation system as measured by counting the relative number of visual predicates the subjects used in a small description of their experience in the VE. This could be explained by the fact that the VE was mainly visual. A higher proportion of auditory predicates, indicating a preference for the auditory representation system, resulted in significantly lower presence. Use of the kinesthetic representation system showed a positive significant correlation with presence when a virtual body was used and a negative significant correlation when no virtual body was used. The level of presence also increased with the first perceptual position. To determine whether preference of predicates determined presence or the other way around, a second experiment was performed with six subjects where the subject had to write a report before they entered the VE. The regression model based on these results did not successfully predict presence. Slater et al. ${ }^{11}$ performed another experiment with 24 subjects where the representation system and perceptual position preferences were measured using a Likert-scale questionnaire filled in by the subjects prior to immersion in the VE. The level of presence was positively significantly associated with $\mathrm{V}$ and $\mathrm{K}$ (a virtual body was included in the VE), and negatively associated with A.

Slater et al., ${ }^{42}$ in a later study already described in the section on vividness, found that including dynamic shadows only increased presence when the subject had a preference for the visual representation system as measured by an updated version of the questionnaire used in the previous experiment.

Bangay and Preston ${ }^{8}$ measured presence at a public VR ride. Two age groups were represented in greater number: between ages 10 and 20 and between ages 35 and 45 . The older age group "tends to provide lower scores for immersion and excitement consistently."

\section{Conclusions on causes of presence}

The empirical research on the causes of presence, although sometimes not consistent, has 
provided more insight into which factors are important for creating presence. On all three types of causes, vividness, interactivity, and user characteristics, empirical evidence has been found for the influence of several causes. The construct presence however was often operationalized by questionnaires, which were not proven reliable or valid. Conclusions on the causes of presence therefore have to be taken with some precaution. For further research the use of valid and reliable presence questionnaires like the IPQ or ITC-SOPI, is recommended. Other important questions on the causes of presence remain as well, the most prominent ones being perhaps on the interaction between the different causes, and which factors have the biggest influence.

Knowledge of the causes of presence is valuable for effective and efficient design of VEs. However, especially on the subject of interaction and interactivity, more research is needed.

\section{DISCUSSION}

There are still major gaps in the knowledge related to presence. One important shortcoming for therapeutic applications is the lack of conclusive research on the relationship between presence, often only measured using questionnaires, and emotional responses such as fear. On top of this the questionnaires used to measure presence are often not reliable nor validated.

Furthermore, there is still not sufficient information on which aspects of the VR system contribute to presence to support the design process of therapeutic applications. The role of aspects of the human-computer interaction is especially unclear.

In general the research reviewed in this paper has been found to be insufficient to determine the effect of VE parameters on the effectiveness of VR as a tool in psychological therapy. Probably the concept of presence alone will not be sufficient to bridge this gap, arguing for the identification of other constructs that play a role in VR experiences. There is more to VR than presence.

\section{REFERENCES}

1. Lombard, M., \& Ditton, T. (1997). At the heart of it all: The concept of presence. Journal of Computer-Mediated Communication, 3(2).

2. Lombard, M., \& Ditton, T. (2000). Measuring presence: A literature-based approach to the development of a standardized paper-and-pencil instrument. Paper presented at the Presence 2000 Workshop, March 27-28, Delft.

3. Sheridan, T.B. (1992). Musings on telepresence and virtual presence. Presence, 1:120-126.

4. Heeter, C. (1992). Being there: The subjective experience of presence. Presence, 1:262-271.

5. Schloerb, D.W. (1995). A quantitative measure of telepresence. Presence, 4:64-80.

6. Slater, M., \& Wilbur, S. (1997). A framework for immersive virtual environments (FIVE): Speculations on the role of presence in virtual environments. Presence, 6:603-616.

7. Zeltzer, D. (1992). Autonomy, interaction, and presence. Presence, 1:127-13.

8. Bangay, S., \& Preston, L. (1998). An investigation into factors influencing immersion in interactive virtual environments. In: Riva, G., Wiederhold, B.K., Molinari, E., (eds.) Virtual environments in clinical psychology and neuroscience. Amsterdam: Ios Press.

9. Witmer, B.G., \& Singer, M.J. (1998). Measuring presence in virtual environments: A presence questionnaire. Presence, 7:225-240.

10. Lombard, M. (2000, September). Resources for the study of presence: Presence explication. Retrieved September 3, 2000 from the World Wide Web: http://nimbus.temple.edu/ mlombard/Presence/ explicat.htm.

11. Slater, M., Usoh, M., \& Steed, A. (1994). Depth of presence in virtual environments. Presence, 3:130-144.

12. Biocca, F. (1997). The Cyborg's dilemma: Progressive embodiment in virtual environments. Journal of Computer-Mediated Communication, 3(2).

13. Slater, M., \& Steed, A. (2000). A virtual presence counter. Presence, 9:413-434.

14. Flach, J.M., \& Holden, J.G. (1998). The reality of experience: Gibson's way. Presence, 7:90-95.

15. Schuemie, M.J., \& van der Mast, C.A.P.G. (1999). Presence: Interacting in virtual reality? Proceedings of the Twentieth Workshop on Language Technology 15, May 19-21, 1999, Enschede, Netherlands.

16. Zahorik, P., \& Jenison, R.L. (1998). Presence as beingin-the-world. Presence, 7:78-89.

16a. Heidigger, M. (1962). Being and time (Macquarie J, Robinson E, transl.) San Francisco: HarperCollins.

17. Gibson, J.J. (1979). The ecological approach to visual perception. Boston: Houghton Mifflin Co.

18. Mantovani, G., \& Riva, G. (1999). “Real” presence: How different ontologies generate different criteria for presence, telepresence, and virtual presence. Presence, 8:540-550.

19. O'Brien, J. Büscher, M., Rodden, T., \& Trevor, J. (1998). 
"Red is behind you": The experience of presence in shared virtual environments. Paper presented at the Workshop on Presence in Shared Virtual Environments.

20. Sheridan, T.B. (1999). Descartes, Heidegger, Gibson, and God: Towards an eclectic ontology of presence. Presence, 8:551-559.

21. Schubert, T.W., Friedman, F., \& Regenbrecht, H.T. (1999). Embodied presence in virtual environments. In: Paton R, Neilson I (Eds.). Visual representations and interpretations. Springer-Verlag, London, pp. 268-278.

22. Prothero, J.D., Parker, D.E., \& Furness, T., III. (1995). Towards a robust, quantitative measure for presence. Proceedings of the Conference on Experimental Analysis and Measurement of Situation Awareness, pp. 359-366.

23. Kalawsky, R. (2000). The validity of presence as a reliable human performance metric in immersive environments. Paper presented at the Presence 2000 Workshop.

24. Lackner, J.R., \& DiZio, P. (1998). Spatial orientation as a component of presence: Insights gained form nonterrestrial environments. Presence, 7:108-115.

25. Slater, M., Pertaub, D., \& Steed, A. (1999). Public speaking in virtual reality: Facing an audience of avatars. IEEE Computer Graphics and Applications, 19:6-9.

26. Welch, R.B. (1999). How can we determine if the sense of presence affects task performance? Presence, 8:574577.

27. Ellis, S.R. (1996). Presence of mind: A reaction to Thomas Sheridan's "Further musing on the psychophysics of presence." Presence, 5:247-259.

28. Mania, K., \& Chalmers, A. (2000). A user-centered methodology for investigating presence and task performance. Paper presented at the Presence 2000 Workshop, March 27-28, Delft.

29. Kim, T., \& Biocca, F. (1997). Telepresence via television: Two dimensions of telepresence may have different connections to memory and persuasion. Journal of Computer-Mediated Communication, 3(2).

30. Slater, M., Sagadic, A., Usoh, M., \& Schroeder, R. (1998). Small group behaviour in a virtual and real environment: A comparative study. BT Presence Workshop, Martlesham Heath, UK, June 11-12.

31. Steed, A., Slater, M., \& Sadagic, A. (1999). Leadership and collaboration in shared virtual environments. Proceedings of the IEEE Virtual Reality 1999 conference, Houston, March 1999, pp. 112-115.

32. Hodges, L., Rothbaum, B.O., Kooper, R., Opdyke, D., Meyer, T., de Graaf, J.J., \& Williford, J.S. (1994). Presence as the defining factor in a VR application. Technical Report GIT-GVU-94-5, Georgia Institute of Technology.

33. Regenbrecht, H.T., Schubert, T.W., \& Friedman, F. (1998). Measuring the sense of presence and its relation to fear of heights in virtual environments. International Journal of Human-Computer Interaction. 10:233249.

34. Schuemie, M.J., Bruynzeel, M., Drost, L., Brinckman, M., de Haan, G., Emmelkamp, P.M.G., \& van der Mast, C.A.P.G. (2000). Treatment of acrophobia in virtual reality: A pilot study. In: Broeckx, F., Pauwels, L. (eds.) Conference Proceedings Euromedia 2000. May 8-10, Antwerp, Belgium, pp. 271-275.

35. North, M.M., North, S.M., \& Coble, J.R. (1998). Virtual reality therapy: An effective treatment for phobias. Virtual Environments in Clinical Psychology and Neuroscience. Amsterdam: IOS Press.

36. Freeman, J., Avons, S.E., Meddis, R., Pearson, D., \& IJsselsteijn, W. (2000). Using behavioral realism to estimate presence: A study of the utility of postural responses to motion stimuli. Presence, 9:149-164.

37. Slater, M., Steed, A., \& Usoh, M. (1993). The virtual treadmill: A naturalistic metaphor for navigation in immersive virtual environments. In: Goebel, M. (ed.), First Eurographics Workshop on Virtual Reality Environments, Polytechnical University of Catalonia, Sept. 7, 1993, pp. 71-83.

38. Towell, J., \& Towell, E. (1997). Presence in text-based networked virtual environments or "MUDS." Presence, 6:590-595.

39. Dinh, H.Q., Walker, N., \& Hodges, L. (1999). Evaluating the importance of multi-sensory input on memory and the sense of presence in virtual environments. Proceedings of the IEEE Virtual Reality 1999 Conference, Houston, March 1999, pp. 222-228.

40. Hendrix, C., \& Barfield, W. (1996). Presence within virtual environments as a function of visual display parameters. Presence, 4:274-289.

41. Usoh, M., Catena, E., Arman, S., \& Slater, M. (2000). Using presence questionnaires in reality. Presence, in press.

42. Slater, M., Usoh, M., \& Chrysanthou, Y. (1995). The influence of dynamic shadows on presence in immersive virtual environments. Virtual Environments 95, 8-21.

43. Slater, M. (1999). Measuring presence: A response to the Witmer and Singer Presence Questionnaire. Presence, 8:560-565.

44. Schubert, T.W., Friedmann, F., \& Regenbrecht, H.T. (1999). Decomposing the sense of presence: Factor analytic insights. Presented at the 2nd International Workshop on Presence, University of Essex, UK, 6-7 April 1999.

45. Lessiter, J., Freeman, J., Keogh, E., \& Davidoff, J. (2000). Development of a new cross-media presence questionnaire: The ITC-Sense of presence. Paper presented at the Presence 2000 Workshop, March 27-28, Delft.

46. Freeman, J., Avons, S.E., Pearson, D., \& IJsselsteijn, W. (1999). Effects of sensory information and prior experience on direct subjective ratings of presence. Presence, 8:1-13.

47. Welch, R.B., Blackmon, T.T., Liu, A., Mellers, B., \& Stark, L.W. (1996). The effects of pictoral realism, delay of visual feedback, and observer interactivity on the subjective sense of presence. Presence, 5:263-273.

48. IJsselsteijn, W.A., \& de Ridder, H. (1998). Measuring temporal variations in presence. Paper presented at the Presence in Shared Virtual Environments Workshop, University College, London, 10-11 June, 1998.

49. Freeman, J., \& Avons, S.E. (2000). Focus group exploration of presence through advanced broadcast 
services. Proc. of the SPIE, Human Vision and Electronic Imaging, pp. 3959-3976.

50. Sheridan, T.B. (1996). Further musings on the psychophysics of presence. Presence, 5:241-246.

51. Meehan, M. (2000). An objective surrogate for presence: Physiological response. Paper presented at the Presence 2000 Workshop, March 27-28, Delft.

52. Wiederhold, B.K., Davis, R., \& Wiederhold, M.D. (1998). The effect of immersiveness on physiology. Virtual Environments in Clinical Psychology and Neuroscience. Amsterdam: IOS Press.

53. Wilson, G., \& Sasse, M.A. (2000). The head or the heart? Measuring the impact of media quality. Extended Abstracts of CHI 2000, April 1-6, The Hague, The Netherlands, pp. 117-118.

54. Slater, M., \& Usoh, M. (1993). Representation systems, perceptual positions, and presence in immersive virtual environments. Presence, 2:221-233.

55. Steuer, J. (1992). Defining virtual reality: Dimensions determining telepresence. Journal of Communication, 42(4):72-93.

56. Axelsson, A., Abelin, A., Heldal, I., Schroeder, R., \& Wideström, J. (2000). Cubes in the cube; A comparison of collaboration in virtual and real environments. Paper presented at the Presence 2000 Workshop, March 27-28, Delft.

57. Barfield, W., Baird, K.M., \& Bjorneseth, O.J. (1998). Presence in virtual environments as a function of type of input device and display update rate. Displays, 19:91-98.

58. Prothero, J.D., \& Hoffman, H.G. (1995). Widening the field of view increases the sense of presence in immersive virtual environments. Technical Report TR-952, Human Interface Technology Lab.

59. Prothero, J.D., Hoffman, H.G., Parker, D.E., Furness, T.A., III, \& Wells, M.J. (1995). Foreground/background manipulations affect presence. Proceedings of the Human Factors and Ergonomics Society 39th Annual Meeting.

60. Schubert, T.W., Regenbrecht, H.T., \& Friedman, F. (2000). Real and illusory interaction enhance presence in virtual environments. Paper presented at the Presence 2000 Workshop, March 27-28, Delft.

61. Gilkey, R.H., \& Weisenberger, J.M. (1995). The sense of presence for the suddenly deafened adult. Presence, 4:357-361.

62. Hendrix, C., \& Barfield, W. (1996). The sense of presence within auditory virtual environments. Presence, 5:290-301.

63. Hoffman, H., Groen, J., Rousseau, S., Hollander, A., Winn, W., Wells, M., \& Furness, T. (1996). Tactile augmentation: Enhancing presence in virtual reality with tactile feedback from real objects. Paper presented at the meeting of the American Psychological Society, San Francisco, CA.

64. Slater, M., Steed, A., McCarthy, J., \& Maringelli, F., (1998). The influence of body movement on subjective presence in virtual environments. Human Factors, 40:469-477.

65. Thie, S., \& van Wijk, J. (1998). A general theory on presence: Experimental evaluation of social virtual presence in a decision making task. Paper presented at the Presence in Shared Virtual Environments Workshop. University College London, 10-11 June 1998.

66. Hoffman, H., Prothero, J., Wells, M., \& Groen, J. (1998). Virtual chess: Meaning enhances users' sense of presence in virtual environments. International Journal of Human-Computer Interaction, 10(3):251-263.

Address reprint requests to: Martijn J. Schuemie M.Sc. Department of Information Systems and Software Engineering Faculty of Information Technology and Systems Delft University of Technology Zuidplantsoen 4 2628 BZ, Delft, The Netherlands E-mail: m.j.schuemie@its.tudelft.nl 